\title{
Compact high gain and wideband octagon microstrip Yagi antenna
}

\begin{abstract}
A new structure of microstrip Yagi antenna is proposed in this article in order to obtain a high gain and wide bandwidth. It is called Octagon Microstrip Yagi Antenna. The Octagon Microstrip Yagi Antenna has been simulated, optimized, constructed, and tested. According to simulated results, it appears that the simulated Octagon Microstrip Yagi Antenna has the ability to obtain $15.97 \%$ bandwidth and gain of $11.16 \mathrm{~dB}$ in a compact size of $70 \mathrm{~mm} \times 75$ $\mathrm{mm}$. On the other hand, experimental results show that the fabricated Octagon Microstrip Yagi Antenna can achieve a gain of $11 \mathrm{~dB}$ with $13.8 \%$ bandwidth. It can be seen that there is a good agreement between simulation and measurement results. Hence, it gives evidence that the proposed antenna is capable of producing a high gain with a wide bandwidth in a smaller size as compared to the others.
\end{abstract}

Keyword: High gain; Wideband; Microstrip Yagi antenna; ISM band frequency of 5.8 $\mathrm{GHz}$ 